\title{
Seventeen years of experience with ATRA-based therapy for acute promyelocytic leukaemia: Long-term follow-up of patients treated at S. Martino Hospital, Genoa
}

\author{
MARINO CLAVIO, ANNA GHISO, CHIARA GHIGGI, MAURO SPRIANO, NICOLETTA COLOMBO, \\ RAFFAELLA GRASSO, RICCARDO VARALDO, MAURIZIO MIGLINO, IVANA PIERRI, \\ FRANCESCA OLCESE, SARA AQUINO, SAMANTHA BIASCO, ENRICO BALLEARI, \\ ANGELO MICHELE CARELLA, MARIO SESSAREGO and MARCO GOBBI
}

Department of Haematology and Oncology, S. Martino Hospital and the University of Genoa, Viale Benedetto XV, N 6, 16132 Genoa, Italy

Received October 27, 2008; Accepted December 22, 2008

DOI: $10.3892 /$ or_00000322

\begin{abstract}
We conducted a long-term follow-up retrospective study on 91 consecutive newly diagnosed acute promyelocytic leukaemia (APL) patients. Induction and consolidation therapy were well-tolerated by most patients. Of the 79 patients who were initially treated with the all-trans retinoic acid (ATRA)-containing regimens, there were 3 haemorrhagic deaths during the first period of therapy (4\%) and one in consolidation which was due to infection. Following consolidation, molecular assessment of response was performed on 67 patients, and 66 were found to have achieved cytogenetic and molecular remission (98\%). After a median follow-up of 100 months (12-192), 10 of the 75 patients who achieved complete remission (13\%) relapsed. Seventy-eight percent of the patients were expected to be alive at 14 years from diagnosis, i.e., 90 and $48 \%$ of patients of intermediate-low risk and high risk at presentation, respectively $(\mathrm{p}=0.0009)$. Sixty-nine patients were in molecular remission after firstline and/or salvage therapy (74\%). To date, 4 patients out of the 91 have undergone salvage allogeneic transplant (4\%).
\end{abstract}

\section{Introduction}

The treatment of acute promyelocytic leukaemia has been based on all-trans retinoic acid (ATRA) for more than 15 years (1-7). Several studies have been published involving

Correspondence to: Dr Marino Clavio, Department of Haematology, University of Genoa, Viale Benedetto XV, N 6, 16132 Genoa, Italy

E-mail: claviom@unige.it

Key words: all-trans retinoic acid, PML-RAR $\alpha$, acute promyelocytic leukaemia, fluorescent in situ hybridization different therapeutic approaches, and currently, there is a tendency to design risk-adapted strategies according to a predefined risk of relapse (8). However, there have been few comprehensive reports on long-term experience since the introduction of ATRA to the therapeutic armamentarium of APL.

We, therefore, performed a retrospective study on the clinical and haematological features at presentation, as well as on the management and long-term outcome of 91 consecutive, newly diagnosed APL patients who were treated and followedup over a 17-year period at the Department of Haematology and Oncology, S. Martino Hospital, Genoa, Italy.

\section{Patients and methods}

Diagnosis. The diagnosis of possible APL was established on the basis of clinical presentation and morphological FAB criteria (9). Cytogenetic demonstration of $t(15 ; 17)$ and or detection by fluorescent in situ hybridization (FISH) or RT-PCR of PML-RAR $\alpha$ was required to confirm the diagnosis (10). Patients with morphological diagnosis alone of APL were excluded from the study.

Karyotyping was performed on 24-h cultured bone marrow cells according to standard procedures. At least 20 metaphases were analysed.

FISH analysis was performed using LSI PML/RAR $\alpha$ Dual Color Dual Fusion translocation probe (Vysis, Inc., Downers Grove, IL); at least 100 cells were analysed.

Clinical and haematological features at diagnosis of the entire series of patients were reviewed. Response to therapy, disease-free survival (DFS) and overall survival (OS) were only analysed for patients who had initially been treated with ATRA-containing protocols.

We analysed serial bone marrow aspirates by RT-PCR to detect PML/RAR-rearrangements. Samples were examined at diagnosis, then again after achieving complete clinical remission (CR), and periodically thereafter.

CR requirements included: no clinical evidence of APL, haemoglobin $>10 \mathrm{~g} / \mathrm{dl}$ without transfusion, neutrophil count 
Table I. Clinical and haematological features of patients.

\begin{tabular}{ll}
\hline Number of patients & 91 \\
Gender (male/female) & $45 / 46$ \\
Median age (range) & $41(17-83)$ \\
FAB subtypes: & \\
M3 & $82(90 \%)$ \\
M3v & $9(10 \%)$ \\
De novo APL & $88(97 \%)$ \\
Secondary APL & $3(3 \%)$ \\
to NHL & 1 \\
to breast cancer & 2 \\
Available/studied karyotypes with conventional & $91(100 \%)$ \\
cytogenetic and FISH & \\
Presence of t(15;17) & $91(100 \%)$ \\
Detection of t(15;17) and +8 & $4(4 \%)$ \\
Patients with muco-cutaneous haemorrhagic signs & $82(90 \%)$ \\
Major haemorrhagic episodes & $9(10 \%)$ \\
Cerebral haemorrhage & 4 \\
Retinal haemorrhage & 3 \\
Gastrointestinal haemorrhage & 1 \\
Metrorrhagia & 1 \\
Patients with clinical or laboratory evidence of DIC & $80(88 \%)$ \\
\hline
\end{tabular}

M3v, M3 microgranular variant; DIC, disseminated intravascular coagulation.
Table II. Clinical and haematological features of patients at diagnosis.

\begin{tabular}{ll}
\hline Haematologic risk: & \\
Low (LR) & $28(31 \%)$ \\
Intermediate (IR) & $41(45 \%)$ \\
High (HR) & $22(24 \%)$ \\
Median Hb at diagnosis, g/dl (range) & $8.8(4.5-14)$ \\
Median WBC at diagnosis, x109/1 (range) & $3(0.5-153)$ \\
Median PLT at diagnosis, x10 $6 / 1$ (range) & $24(5-278)$ \\
Median serum LDH, U/l (range) & $509(207-4936)$ \\
Median fibrinogen, mg/dl (range) & $194(64-538)$ \\
Median XDP, $\mu$ g/l (range) & $1414(56-6400)$ \\
Co-morbidity: & \\
Hypertension & $16(18 \%)$ \\
Diabetes & $3(3 \%)$ \\
Ischaemic cardiopathy & $2(3 \%)$ \\
Heart failure & 1 \\
Patients who died before therapy & $3(3 \%)$ \\
Patients treated with ATRA-containing protocols & $79(87 \%)$ \\
AIDA LAP 0493 & $51(65 \%)$ \\
AIDA 2000 & $18(23 \%)$ \\
MY-AIDA & $10(12 \%)$ \\
\end{tabular}

Patients not receiving ATRA-containing protocols 9 (10\%)

Haematologic risk in patients treated with

ATRA-containing protocols:

$\begin{array}{ll}\text { Low (LR) } & 26(33 \%) \\ \text { Intermediate (IR) } & 34(43 \%) \\ \text { High (HR) } & 19(24 \%)\end{array}$

$>1.5 \times 10^{9} / 1$, platelets $>100 \times 10^{9} / 1$, and normally cellular or moderately hypocellular bone marrow with $<5 \%$ promyelocytes and normalization of chromosome analysis and FISH for PML/RAR $\alpha$.

Molecular response was defined as undetectability of PML-RAR $\alpha$ transcript by RT-PCR.

The Kaplan-Meier method was used to estimate the probability of disease-free and overall survival, while the Log-rank test was used to compare DFS and OS duration among different groups.

Clinical and haematological features. Between January 1991 and July 2007, APL was diagnosed in 91 patients at our centre. The clinical and haematological features of this cohort of patients are provided in Tables I and II.

In brief, the median age of patients was 41 years (range 17-83). Seventy-five patients were $<60$ years of age, and 16 were $\geq 60$ years of age. FAB subtypes were found to be M3 in 82 patients $(90 \%)$ and M3v in 9 patients $(10 \%)$.

Typical $\mathrm{t}(15 ; 17)$ was observed in all patents, while trisomy 8 , as an additional cytogenetic alteration, was detected in 4 patients.

The vast majority of patients had de novo APL. One patient developed APL 3 years after chemotherapy for NHL, while 2 other patients became affected 3 years after radiochemotherapy for breast carcinoma.
Mucosal or cutaneous bleeding was present in the majority of patients (90\%), but severe haemorrhagic syndromes were only observed in 9 patients $(10 \%)$, involving the brain $(n=4)$, the retina $(n=3)$, and the gastrointestinal tract $(n=1)$ and bladder $(n=1)$. Cerebral haemorrhages were always fatal.

Clinical manifestations and laboratory changes related to disseminated intravascular coagulation (DIC) were observed in 80 patients $(88 \%)$.

Prognostic evaluation was performed according to the PETHEMA scoring system (8). The risk was low in 28 patients $(31 \%)$, intermediate in $41(45 \%)$ and high in 22 (24\%).

Induction regimens. As shown in Fig. 1, 3 patients died of brain haemorrhage soon after admission before therapy. Nine patients were not initially treated with ATRA, since the drug was not available at the time. Thus, they received a combination of idarubicin $(n=4)$ or daunorubicin $(n=5)$ plus Ara-C. Four patients were treated with ATRA-containing regimens due to relapse or refractory disease. Two of these patients are alive and disease-free at 187 and 215 months from diagnosis. A third patient died of myocardial infarction at 70 months post-treatment while in molecular remission. The fourth died of disease. 


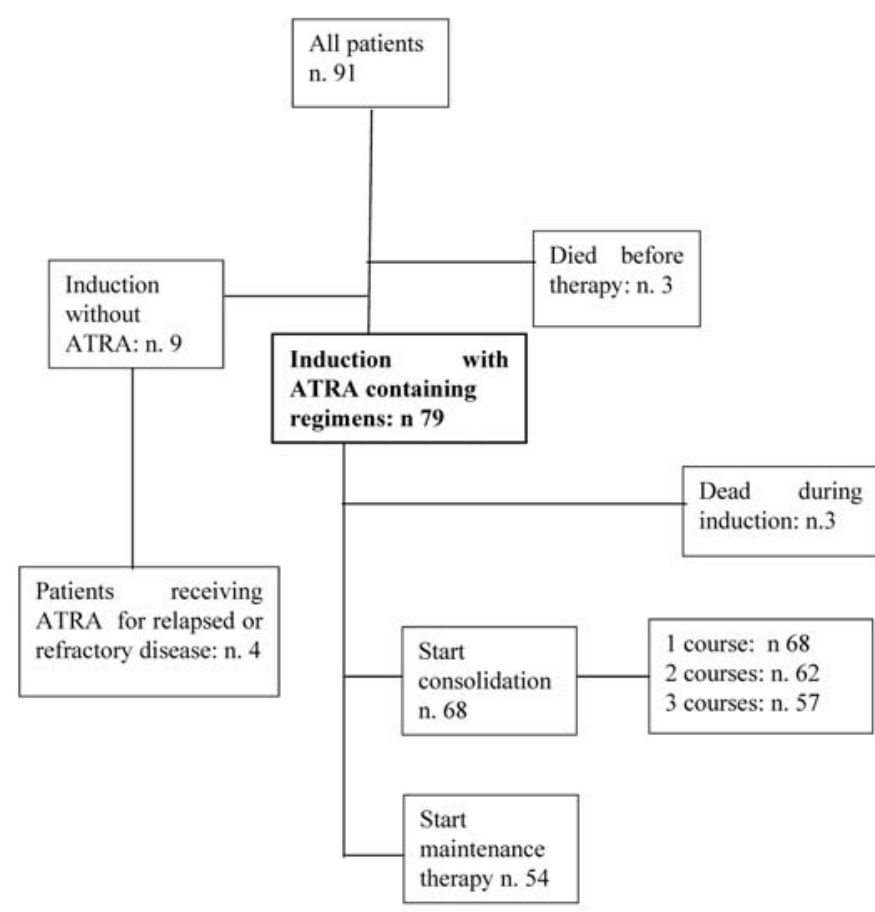

Figure 1. Treatment of acute promyelocytic leukaemia patients. ATRA, alltrans retinoic acid.

Five patients never received ATRA. Two of them underwent allogeneic transplant and are in molecular remission at 172 and 184 months. Two patients died of disease at 16 and 12 months. A third patient committed suicide while in CR at 7 months after the end of therapy.

Seventy-nine patients were enrolled in multi-centre GIMEMA trials (51 in AIDA LAP 0493, 18 in AIDA 2000, 10 in MY-AIDA ) $(2,7)$.

Induction for all of these protocols consisted in administering $45 \mathrm{mg} / \mathrm{m}^{2} /$ day of oral ATRA from day 1 , associated with $12 \mathrm{mg} / \mathrm{m}^{2}$ of intravenous IDA on days 2, 4, 6 and 8 .

A 26-year-old pregnant woman with low risk APL started induction therapy with ATRA alone, achieving haematological remission after 35 days of therapy. After delivery she was administered conventional consolidation courses and received ATRA-containing maintenance therapy for 2 years. The patient still maintains molecular response 7 years after diagnosis.

Consolidation and maintenance therapy. Patients enrolled in GIMEMA trials received consolidation and maintenance therapy as per the specific protocols $(2,7)$. Only patients receiving ATRA-containing regimens were analyzed for response to therapy, toxicity, DFS and OS.

\section{Results}

Haematological recovery, toxicity and response to induction. As indicated in Table III, induction therapy was welltolerated by most patients. Of the 79 consecutive induction courses, there were 3 deaths during the first period of therapy (4\%), which were caused by haemorrhagic and thrombotic complications related to DIC and thrombocytopenia.
Table III. Haematologic recovery, toxicity and response to induction in 79 patients treated with ATRA-containing protocols.

$\begin{array}{lc}\text { Days with neutrophils }<0.5 \times 10^{9} / 1 \text { (range) } & 6(0-20) \\ \text { Days with neutrophils }<1 \times 10^{9} / 1 \text { (range) } & 8(1-26) \\ \text { Days with platelets }<25 \times 10^{6} / 1 \text { (range) } & 15(1-21) \\ \text { Days with platelets }<50 \times 10^{6} / 1 \text { (range) } & 18(6-25) \\ \text { Median of RBC units (range) } & 7(1-15) \\ \text { Median of PLT units (range) } & 6(1-15) \\ \text { Median of plasma units (range) } & 6(0-18) \\ \text { Median length of first hospitalisation, days (range) } & 30(14-56) \\ \text { Patients treated with G-CSF } & 11 \\ \text { Median number of G-CSF vials (range) } & 6(2-13) \\ \text { Median number of days with fever (range) } & 5(0-14) \\ \text { Median number of days with i.v. antibiotic } & 14(0-32) \\ \text { therapy (range) } & \\ \text { Patients who died during induction therapy } & 3(4 \%) \\ \text { Brain haemorrhage } & 1 \\ \text { DIC } & 1 \\ \text { Myocardial infarction } & 1 \\ \text { Retinoic acid syndrome (RAS) }\end{array}$

Complications:

Fever of unknown origin $\quad 15$

Sepsis $\quad 7$

Pneumonia $\quad 5$

Oral infections $\quad 3$

Retinal haemorrhage $\quad 1$

Acute renal failure $\quad 1$

Pericarditis 1

Pleuritis 2

Gastrointestinal symptoms $\quad 19$

Patients evaluated for haematological and $\quad 76$ karyotypic response

Patients in CR

$75 / 76(99 \%)$

Patients evaluated for molecular response $\quad 61$

Patients with molecular response $\quad$ 47/61 (62\%)

RBC units, red blood cell units; PLT units, platelet units; G-CSF, granulocyte-stimulating factor.

Twenty-five patients (42\%) experienced either grade 3 or 4 leukopenia and thrombocytopenia. Neutropenia $\left(\mathrm{N}<0.5 \times 10^{9} / 1\right)$ and thrombocytopenia (Plt $\left.<25 \times 10^{9} / 1\right)$ lasted a median of 6 days (range 1-21) and 15 days (range 2-17) from the end of anthracycline therapy, respectively. Patients received a median of 7 and 6 packed erythrocyte and platelet units, respectively and 6 units of fresh frozen plasma. G-CSF was administered to 11 patients for a median of 6 days. Twelve major infectious complications were recorded (sepsis in 7 patients, pneumonia in 5) as well as minor infections in 3 cases. Non-haematological toxicity was very mild.

Symptoms of retinoic acid syndrome (RAS) were reported in 8 patients $(10 \%)$ leading to permanent discontinuation of 
Table IV. Haematologic recovery, toxicity and molecular response to consolidation courses.

\begin{tabular}{ll}
\hline Patients receiving first consolidation course & $68 / 76(89 \%)$ \\
Patients receiving second consolidation course & $62 / 76(82 \%)$ \\
Patients receiving third consolidation course & $57 / 76(75 \%)$ \\
Reasons for not receiving complete consolidation: & \\
Infection & 8 \\
Relapse & 3 \\
Toxicity, age and other reasons & 10 \\
Days with neutrophils $<0.5 \times 10^{9} / 1$ (range) & $5(0-15)$ \\
Days with neutrophils $<0.1 \times 10^{9} / 1$ (range) & $7(0-17)$ \\
Days with platelets $<25 \times 10^{6} / 1$ (range) & $6(0-15)$ \\
Days with platelets $<50 \times 10^{6} / 1$ (range) & $7(0-17)$ \\
Median of RBC units (range) & $1(0-4)$ \\
Median of PLT units (range) & $1(0-3)$ \\
Infectious complications: & \\
Septic shock (lethal) & 1 \\
Sepsis and pneumonia & 3 \\
Fever of unknown origin & 14 \\
Patients evaluated for molecular response & 67 \\
Molecular response & $66 / 67(98 \%)$ \\
\hline
\end{tabular}

RBC units, red blood cell units; PLT units, platelet units.

ATRA therapy in 2 cases and to temporary discontinuation in 2 patients.

Seventy-six of the 79 patients $(96 \%)$ were evaluated for response, and CR was achieved by 75 patients (95\%). Sixtyone patients underwent molecular evaluation of response at the end of induction, and 47 (62\%) achieved molecular remission.

Haematological recovery, toxicity and response to the consolidation courses. Table IV provides details regarding the compliance of patients to consolidation therapy as well as haematological recovery, support therapy and complications. Fifty-seven $(75 \%)$ patients completed the 3 consolidation courses.

In brief, we recorded less severe myelotoxicity during consolidation therapy than in induction. Twenty percent of the patients presented fever of unknown origin. Sepsis due to Staphylococcus aureus complicated by septic shock was diagnosed in a young patient who had already achieved CR, and eventually resulted in the death of the patient. The need for transfusional support was milder than after the induction course.

All patients maintained haematological response at the end of consolidation.

Following consolidation, molecular assessment of the response was performed on 67 patients, and 66 were found to have achieved cytogenetic and molecular remission (98\%).

Maintenance therapy. Details of maintenance therapy are provided in Table V. Fifty-four patients $(68 \%)$ completed maintenance therapy. Twenty-five patients received ATRA (45 mg/m²/day for 15 days every 3 months), 20 were admini-
Table V. Maintenance therapy.

\begin{tabular}{ll}
\hline Patients completing maintenance therapy & 54 \\
ATRA & $25(46 \%)$ \\
ATRA + chemotherapy & $20(37 \%)$ \\
Gemtuzumab ozogamicin (GO-Mylotarg) & $3(6 \%)$ \\
Chemotherapy & $6(11 \%)$ \\
Patients not receiving maintenance therapy & 25 \\
Reasons: & 5 \\
Death during induction or consolidation & 8 \\
Toxicity & 4 \\
Relapse & 1 \\
Too early & 6 \\
Randomisation of protocol & 1 \\
Decision of patient or clinician & 54 \\
Patients evaluable for molecular response at the & \\
end of maintenance therapy & $54(100 \%)$ \\
Molecular response &
\end{tabular}

stered ATRA plus chemotherapy, 6 underwent chemotherapy and 3 were administered GO. Therapy with ATRA alone was well-tolerated and showed no significant side effects. Mild myelotoxicity was recorded in patients who underwent other types of maintenance. No severe infections were observed.

Reasons for not receiving maintenance therapy included: death during induction or after first consolidation $(n=5)$, toxicity $(n=8)$, early relapse $(n=4)$, protocol randomisation $(n=6)$ and other reasons $(n=2)$. At the end of maintenance therapy, molecular evaluation of response was performed on 54 patients, all of whom maintained cytogenetic and molecular remission.

Disease-free survival, relapse and overall survival. The median follow-up for living patients was 100 months (10-192).

During follow-up, 10 of the 76 patients who achieved haematologic response relapsed (13\%). Molecular relapse was always associated with haematologic relapse. All relapsing patients showed $\mathrm{t}(15 ; 17)$ and/or PMA-RAR $\alpha$ rearrangement. Two patients presented haematologic relapse associated with central nervous system involvement. Of the relapsed patients, 2 (20\%) had the M3 variant, 4 (40\%) belonged to the high risk group, only 5 had completed consolidation course $(50 \%)$ and $4(40 \%)$ had received maintenance therapy. Table VI lists the clinical data of the relapsed patients. All of these patients were treated with salvage chemotherapy (HDAC, MEC, FLANG, Ara-C plus IDA plus etoposide, GO, ATO). The only patients who achieved haematological and molecular remission were treated with GO and ATO.

Two of the relapsing patients underwent allogeneic BMT from HLA identical related siblings. One of them is alive and disease-free at 7 years from transplant, while the second relapsed one year after transplant and died of disease.

One of the patients who was enrolled in the AIDA 2000 protocol developed a therapy-related myelodysplastic syndrome (RAEB-2) with normal karyotype 6 years later, 
Table VI. Relapses, disease-free and overall survival.

Patients who achieved molecular response 76

Relapsed patients

Median length of CR in relapsed patients, months

$10(2-39)$

Relapses before 4 years

10

FAB subtypes of relapsed patients:

M3

M3v

PETHEMA risk score of relapsed patients:

HR

IR

Administered consolidation courses:

One

$8 / 10(80 \%)$

Two $6 / 10(60 \%)$ $5 / 10(50 \%)$

Patients relapsing during maintenance therapy

$4(40 \%)$

Treatment of relapse:

Chemotherapy

GO

ATO

Allogeneic transplant

No therapy

Median length of CR, months

Median overall survival, months

$76(1-153)$

Patients alive and disease-free:

Among patients initially receiving ATRA

$65 / 79(82 \%)$

In the entire cohort of patients

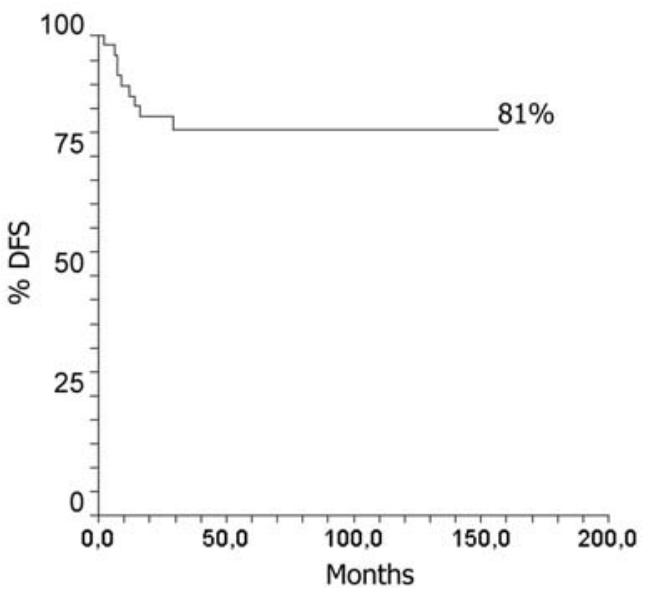

Figure 2. Disease-free survival of patients with acute promyelocytic leukaemia in complete remission after trans retinoic acid-containing therapy. DFS, disease-free survival.

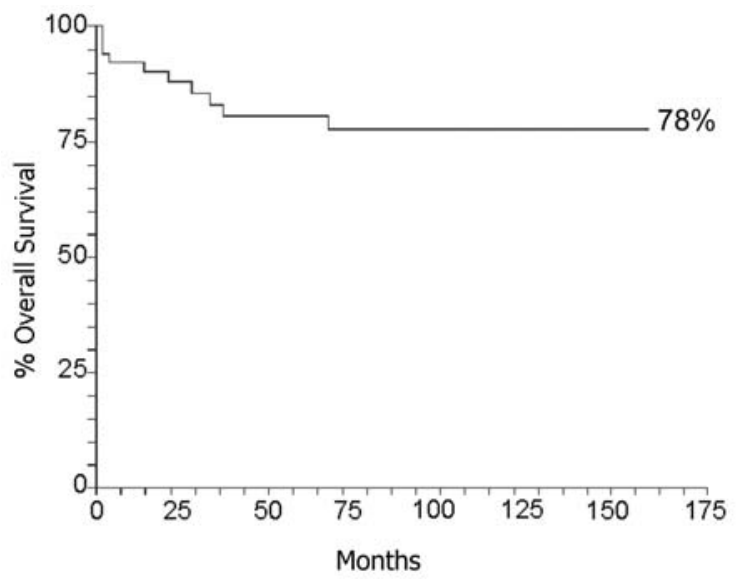

Figure 3. Overall survival in patients with acute promyelocytic leukaemia receiving trans retinoic acid-containing therapy.

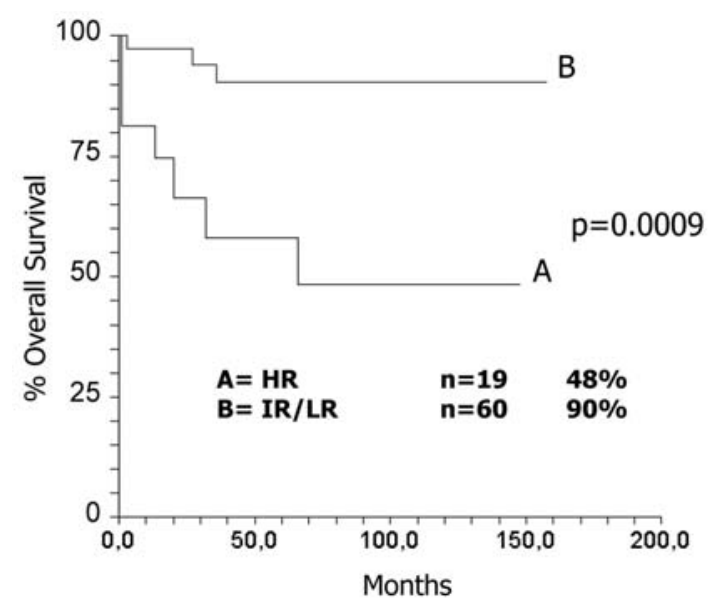

Figure 4. Overall survival in patients with acute promyelocytic leukaemia receiving trans retinoic acid-containing therapy according to haematologic risk at diagnosis. HR, high risk disease; IR, intermediate risk disease; and LR, low risk disease according to the PETHEMA score. and rapidly developed sAML refractory to conventional induction therapy; he received an allogeneic transplant from an unrelated donor and is alive and disease-free 13 months after the transplant. Two other patients died of a second neoplasia, though most likely unrelated to the therapy for APL (renal carcinoma and lung carcinoma, respectively) while in CR for APL.

As shown in Fig. 2, after a median follow-up of 100 months, $81 \%$ of patients achieving CR after ATRA-containing therapy are alive and disease-free at 14 years. Sixty-five of the 79 patients are alive and disease-free while 12 are deceased (3 during induction therapy, 3 of infectious complications during consolidation or salvage therapy, one committed suicide, 5 of disease).

Seventy-eight percent of the patients were expected to be alive at 14 years from diagnosis as reported in Fig. 3. The median survival was 76 months (range 1-151) and the median length of CR was 68 months (range 10-150).

The 3 patients bearing the additional trisomy 8 are alive and disease-free. 
Table VII. Therapy in patients $\geq 60$ years of age.

\begin{tabular}{lc}
\hline Number of patients & 16 \\
FAB subtypes: & \\
M3 & $14(88 \%)$ \\
M3v & $2(12 \%)$ \\
De novo APL & $14(88 \%)$ \\
Secondary APL & $2(12 \%)$ \\
Died during induction & $2 / 16(12 \%)$ \\
Patients evaluated for response & 14 \\
Complete haematologic response & $13 / 14(93 \%)$ \\
Number of administered consolidation courses: & \\
One & $10(71 \%)$ \\
Two & $8(57 \%)$ \\
Three & $6(43 \%)$ \\
Complications: & \\
Pneumonia & 2 \\
Fever of unknown origin & 7 \\
Urinary tract infection & 1 \\
Maintenance therapy & $10(71 \%)$ \\
Patients reaching molecular response & $14 / 14(100 \%)$ \\
Relapsed patients & $1 / 14(7 \%)$ \\
Patients living/deceased & $12 / 4$ \\
Patients alive in molecular response & $12 / 16(75 \%)$ \\
\hline
\end{tabular}

Fig. 4 shows that the expected survival at 14 years is 90 and $48 \%$ in patients with intermediate-low risk and high risk at presentation, respectively ( $\mathrm{p}=0.0009)$.

Eighty-three patients were administered ATRA as initial therapy $(n=79)$ or later on $(n=4)$. Sixty-seven of them $(83 \%)$ are in molecular remission. $(4 \%)$.

To date, 4 patients have undergone allogeneic transplants

Therapy for patients $\geq 60$ years of age. Sixteen patients $(18 \%)$ were 60 years of age or older at diagnosis. Of these, 2 had the M3 variant and 2 had secondary APL.

Two patients died during induction therapy (12\%). With the exception of these cases, the therapeutic strategy was well-tolerated and showed a limited incidence of complications. The first consolidation course was administered to $71 \%$ of these patients, while $57 \%$ were administered two courses and $43 \%$ actually completed the therapeutic programme. Table VII lists the details of therapy. Thirteen of the $14(93 \%)$ patients achieved CR and 12 are alive and disease-free with a median DFS of 57 months.

\section{Discussion}

This study reviews our experience involving APL management over the last 16 years. The percentage of haematologic and cytogenetic response after induction, as well as the long-term molecular results in our series confirm the previously established high efficacy of ATRA-containing therapy in APL patients of all ages $(1-8,11,12)$. Furthermore, our retrospective analysis highlights that achieving a persistently negative $\mathrm{PML} / \mathrm{RAR}$ status as assessed by RT-PCR is associated with favourable DFS and OS $(4,10)$ and that maintenance therapy might be beneficial.

It has been known since the early nineteens that the availability of a molecularly targeted drug has dramatically improved the outcome as shown by the randomised study carried out by the North American Intergroup (13). Our study confirms that although the introduction of ATRA has improved the management of APL coagulopathy and has reduced induction-related deaths, approximately $10 \%$ of patients are still lost on account of severe bleeding (14). There is still a broad area for further studies on transfusional support and management of thrombo-haemorrhagic events.

ATRA-containing regimens show limited side effects. Although myelotoxicity was milder than what is induced by chemotherapy in other AML subtypes, in some patients infectious complications were life threatening events. Thus, in order to reduce the risk, the most recent approaches tailor post-induction therapy to the patient's individual risk $(7,8,12)$.

Non-haematological side effects were negligible and mucositis was mild, even in patients $>60$ years of age and with associated diseases. The good level of tolerability of this therapy is confirmed by the fact that $76 \%$ of patients completed the entire consolidation, and that $83 \%$ of patients received at least two consolidation courses. Therapy may, however, be complicated by RAS, which occurred in $10 \%$ of patients. Our experience confirms that RAS may be severe and can affect the probability of achieving long lasting remission (15). The availability of alternative and equally effective drugs (ATO and GO) (16-18) allowed us to obtain persistent molecular response even in patients who were unable to continue ATRA.

The favourable outcome that was observed upon longterm evaluation in our subgroup of patients aged 60 years or older confirms the good results that have already been reported in this particular subset of patients $(6,11,12)$. On the other hand, the non-optimal feasibility of consolidation therapy indicates the need for post-induction regimens specifically designed for elderly patients. However, these data indicate that age itself is no longer a bad prognostic factor when specific and low toxicity treatments are available.

The relapse rate we observed is lower than what was reported in some larger collaborative trials $(1,2,4-8,11,13)$. Analysis of disease recurrence showed that most relapsed patients belonged to the high risk group, as evaluated by the PETHEMA score (8). However, in our study the risk of relapse was particularly high in patients who did not complete the scheduled consolidation courses. Only $50 \%$ of the relapsed patients in our series had in fact finished the consolidation programme.

On the basis of our therapeutic strategy, patients received appropriate doses of ATRA both during induction and consolidation courses and in many cases even as maintenance therapy. However it was difficult to establish the benefit of maintenance therapy in our cohort of patients. Although maintained patients had a longer DFS than not maintained 
ones (DFS 60 and 44 months, respectively), the series was too small to draw any definitive conclusions, and patient selection biases may have influenced these results. However, previous studies seem to confirm that long-term administration of ATRA may reduce the relapse rate and prolong DFS $(7,8)$.

Applying the quantitative PCR evaluation of PML-RAR $\alpha$ might help to define the indication and the optimal duration of maintenance therapy (19).

It has recently been reported that late relapses (beyond the fourth year after achieving CR) may occur in patients treated with ATRA plus chemotherapy $(20,21)$. To date, after a median follow-up of 100 months, we observed no late relapses, and many patients with persistent molecular response may be cured of their disease.

Only one patient developed post-MDS-AML which may actually have been related to APL therapy. Although the incidence of secondary MDS/AML which has been observed in larger series seems low, this possibility must be taken into consideration (22). Establishing therapeutic programmes that are tailored to individual risk might also reduce the risk of secondary myeloid neoplasms.

As already shown in larger trials, our retrospective study clearly indicates that there is no indication for allogeneic transplant in first CR. This may represent an effective type of salvage treatment for relapsed patients once a second CR has been obtained $(23,24)$.

The inclusion of new specific and selective drugs, such as ATO and GO in front-line therapy, together with improved prognostic characterisation might further improve the quality of response $(12,17,18)$, thus reducing the likelihood of disease recurrence.

\section{References}

1. Fenaux P, Le Deley MC, Castaigne S, Archimbaud E, Chomienne C, Link H, Guerci A, Duarte M, Daniel MT and Bowen D: Effect of all-trans retinoic acid in newly diagnosed acute promyelocytic leukemia. Results of a multicenter randomized trial. Blood 82: 3241-3249, 1993.

2. Avvisati G, Lo Coco F, Diviero D, Falda M, Ferrara F, Lazzarino F, Russo D, Petti MC and Mandelli F: AIDA (all-trans retinoic acid + idarubicin) in newly diagnosed acute promyelocytic leukemia: a Gruppo Italiano Malattie Ematologiche Maligne dell'Adulto (GIMEMA) pilot study. Blood 88: 1390-1398, 1996.

3. Huang M, Yu-Chen Y, Shu-Rong C, Lu MX, Zhoa L, Gu LJ and Wang ZY: Use of all trans retinoic acid in the treatment of acute promyelocytic leukemia. Blood 72: 567-572, 1998.

4. Mandelli F, Diviero D, Avvisati G, Luciano A, Barbui T, Bernasconi C, Broccia G, Cerri R, Falda M, Fioritoni G, Leoni F, Liso V, Petti MC, Rodeghiero F, Saglio G, Vegna ML Visani G, Jehn U, Willemze R, Muus P, Pelicci PG, Biondi A and Lo Coco F: Molecular remission in PML-RAR $\alpha$ positive acute promyelocytic leukemia by combined all-trans retinoic acid and idarubicin (AIDA) therapy. Gruppo Italiano-Malattie Ematologiche Maligne dell'Adulto and Associazione Italiana di Ematologia ed Oncologia Pediatrica Cooperative Groups. Blood 90: 1014-1021, 1997.

5. Sanz MA, Martin G, Rayon C, Esteve J, González M, DíazMediavilla J, Bolufer P, Barragán E, Terol MJ, González JD, Colomer D, Chillón C, Rivas C, Gómez T, Ribera JM, Bornstein R, Román J, Calasanz MJ, Arias J, Alvarez C, Ramos F and Debén G: A modified AIDA protocol with anthracycline-based consolidation results in high antileukemic efficacy and reduced toxicity in newly diagnosed PML-RAR $\alpha$ positive acute promyelocytic leukemia. Blood 94: 3015-3021, 1999
6. Mandelli F, Latagliata R, Avvisati G, Fazi P, Gobbi M, Rodeghiero F, Leoni F, Gobbi M, Nobile F, Gallo E, Fanin R, Amadori S, Vignetti M, Fioritoni G, Ferrara F, Peta A, Giustolisi R, Broccia G, Petti MC, Lo-Coco F; Italian GIMEMA Cooperative Group: Treatment of elderly patients ( $\geq 60$ years) with newly diagnosed acute promyelocytic leukemia. Results of the Italian multicenter group GIMEMA with ATRA and idarubicin (AIDA) protocols. Leukemia 17: 1085-1090, 2003.

7. Lo Coco F, Avvisati G and Vignetti M: Front-line treatment of acute promyelocytic leukemia with AIDA induction followed by risk-adapted consolidation. Results of the AIDA-2000 trial of the Italian GIMEMA group. Blood 104: 115, 2004.

8. Sanz MA, Martin G, Gonzàlez M, Leon A, Rayon C, Rivas C, Colomer D, Amutio E, Capote FJ, Milone GA, De La Serna J, Román J, Barragán E, Bergua J, Escoda L, Parody R, Negri S, Calasanz MJ and Bolufer P: Risk-adapted treatment of acute promyelocytic leukemia with all trans retinoic acid and anthracycline monochemotherapy: a multicenter study by the PETHEMA group. Blood 103: 1237-1243, 2004

9. Bennet JM, Catovsky D, Daniel MT, Flandrin G, Galton DAG, Gralnick HR and Sultan C: Proposals for the classification of acute leukemias. Br J Haematol 33: 451-458, 1976.

10. Lo Coco F, Diviero D, Falini B, Biondi A, Nervi C and Pelicci G: Genetic diagnosis and molecular monitoring in the management of acute promyelocytic leukemia. Blood 94: 12-22, 1999.

11. Sanz MA, Vellenga E, Rayón C, Díaz-Mediavilla J, Rivas C, Amutio E, Arias J, Debén G, Novo A, Bergua J, de la Serna J, Bueno J, Negri S, Beltrán de Heredia JM and Martín G: Alltrans retinoic acid and anthracycline monochemotherapy for the treatment of elderly patients with acute promyelocytic leukemia. Blood 104: 3490-3493, 2004.

12. Sanz MA: Treatment of acute promyelocytic leukemia. Hematology Am Soc Hematol Educ Program pp147-155, 2006.

13. Tallman MS, Andersen JW, Schiffer CA, Appelbaum FR, Feusner JH, Woods WG, Ogden A, Weinstein H, Shepherd L, Willman C, Bloomfield CD, Rowe JM and Wiernik PH: All-trans retinoic acid in acute promyelocytic leukemia: long-term outcome and prognostic factor analysis from the North American Intergroup protocol. Blood 100: 4298-4302, 2002.

14. De la Serna J, Montesinos P, Vellenga E, Rayon C, Parody R, Leon A, Esteve J, Bergua JM, Milone G, Deben G, Rivas C, Gonzalez M, Tormo M, Diaz-Medevilla J, Gonzalez JD, Negri S, Amutio E, Brunet S, Lowenberg B and Sanz MA: Causes and prognostic factors of remission induction failure in patients with acute promyelocytic leukemia treated with all-trans retinoic acid and idarubicin. Blood 111: 3395-3402, 2008

15. De Botton S, Dombret H, Sanz M, Miguel JS, Caillot D, Zittoun R, Gardembas M, Stamatoulas A, Condé E, Guerci A, Gardin C, Geiser K, Makhoul DC, Reman O, de la Serna J, Lefrere F, Chomienne C, Chastang C, Degos L and Fenaux P: Incidence, clinical features and outcome of all-trans retinoid syndrome in 413 cases of newly diagnosed acute promyelocytic leukemia. Blood 92: 2712-2718, 1998.

16. Shen Z-X, Chen G-Q, Ni J-H, Li X-S, Xiong SM, Qiu QY, Zhu J, Tang W, Sun GL, Yang KQ, Chen Y, Zhou L, Fang ZW, Wang YT, Ma J, Zhang P, Zhang TD, Chen SJ, Chen Z and Wang ZY: Use of arsenic trioxide $\left(\mathrm{As}_{2} \mathrm{O}_{3}\right)$ in the treatment of acute promyelocytic leukemia (APL): II. Clinical efficacy and pharmacokinetics in relapsed patients. Blood 89: 3354-3369, 1997.

17. Estey EH, Giles FJ, Beran M, O'Brien S, Pierce SA, Faderl SH, Cortes JE and Kantarjian HM: Experience with gentuzumab ozogamycin ('mylotarg') and all-trans retinoic acid in untreated acute promyelocytic leukemia. Blood 99: 4222-4224, 2002.

18. Estey E, Garcia-Manero G, Ferrajoli A, Faderl S, Verstovsek S, Jones D and Kantarjian H: Use of all-trans retinoic acid plus arsenic trioxide as an alternative to chemotherapy in untreated acute promyelocytic leukemia. Blood 107: 3469-3473, 2006.

19. Santamaria C, Chillon MC, Fernandez C, Martín-Jiménez P, Balanzategui A, García Sanz R, San Miguel JF and González MG: Using quantification of the PML-RARalpha transcript to stratify the risk of relapse in patients with acute promyelocytic leukemia. Haematologica 92: 315-322, 2007.

20. Kelaidi C, Ades L, Chevret S, Sanz M, Guerci A, Thomas X de Botton S, Raffoux E, Rayon C, Fegueux N, Bordessoule D, Rigal-Huguet F, Link H, Stoppa A,Vekhoff A, Meyer-Monard S, Castaigne S, Dombret H, Degos L and Fenaux P: Late first relapses in APL treated with all-trans-retinoic acid- and anthracycline-based chemotherapy: the European APL group experience (APL 91 and APL 93 trials). Leukemia 20: 905-907, 2006. 
21. Latagliata R, Carmosino I, Breccia M, Minni A, Testi A, Iorio N, Lo-Coco F, Avvisati G, Petti MC, Mandelli F and Cimino G: Late relapses in acute promyelocytic leukaemia. Acta Haematol 117: 106-108, 2006.

22. Latagliata R, Petti MC, Fenu S, Mancini M, Spiriti MA, Breccia M, Brunetti GA, Avvisati G, Lo Coco F and Mandelli F: Therapy-related myelodysplastic syndrome - acute myelogenus leukemia in patients treated for acute promyelocytic leukemia: an emerging problem. Blood 99: 822-824, 2002.

23. Thomas X, Dombret H, Cordonnier C, Pigneux A, Gardin C, Guerci A, Vekhoff A, Sadoun A, Stamatoullas A, Fegueux N, Maloisel F, Cahn JY, Reman O, Gratecos N, Berthou C, Huguet F, Kotoucek P, Travade P, Buzyn A, de Revel T, Vilque JP, Naccache P, Chomienne C, Degos L and Fenaux P: Treatment of relapsing acute promyelocytic leukemia by all-trans retinoic acid therapy followed by timed sequential chemotherapy and stem cell transplantation. Leukemia 14: 1006-1013, 2000.
24. Lo Coco F, Romano A, Mengarelli A, Diverio D, Iori AP, Moleti ML, De Santis S, Cerretti R, Mandelli F and Arcese W: Allogenic stem cell transplantation for advanced acute promyelocytic leukemia: results in patients treated in second molecular remission or with molecularly persistent disease. Leukemia 17: 1930-1933, 2003. 